OPEN ACCESS

Edited by:

Harry W. Schroeder,

University of Alabama at Birmingham, USA

Reviewed by:

Leopoldo Flores-Romo,

Cinvestav-IPN, Mexico

Joshy Jacob,

Emory University, USA

Gregory C. Ippolito,

University of Texas at Austin, USA

*Correspondence:

Birgitta Heyman

birgitta.heyman@imbim.uu.se

tShared first authorship.

Specialty section: This article was submitted to B Cell Biology,

a section of the journal

Frontiers in Immunology

Received: 12 October 2016 Accepted: 20 February 2017

Published: 06 March 2017

Citation:

Bergström JJE, XU H and Heyman B (2017) Epitope-Specific Suppression

of IgG Responses by Passively Administered Specific IgG: Evidence of Epitope Masking.

Front. Immunol. 8:238.

doi: $10.3389 /$ fimmu.2017.00238

\section{Epitope-Specific Suppression of IgG Responses by Passively Administered Specific IgG: Evidence of Epitope Masking}

\author{
Joakim J. E. Bergström ${ }^{\dagger}$, Hui Xu ${ }^{\dagger}$ and Birgitta Heyman* \\ Department of Medical Biochemistry and Microbiology, Uppsala University, Uppsala, Sweden
}

Specific IgG, passively administered together with particulate antigen, can completely prevent induction of antibody responses to this antigen. The ability of IgG to suppress antibody responses to sheep red blood cells (SRBCs) is intact in mice lacking FcyRs, complement factor 1q, C3, or complement receptors 1 and 2, suggesting that Fc-dependent effector functions are not involved. Two of the most widely discussed explanations for the suppressive effect are increased clearance of lgG-antigen complexes and/or that IgG "hides" the antigen from recognition by specific B cells, so-called epitope masking. The majority of data on how IgG induces suppression was obtained through studies of the effects on lgM-secreting single spleen cells during the first week after immunization. Here, we show that IgG also suppresses antigen-specific extrafollicular antibody-secreting cells, germinal center B-cells, longlived plasma cells, long-term IgG responses, and induction of memory antibody responses. IgG anti-SRBC reduced the amount of SRBC in the spleens of wild-type, but not of FcyR-deficient mice. However, no correlation between suppression and the amount of SRBC in the spleen was observed, suggesting that increased clearance does not explain IgG-mediated suppression. Instead, we found compelling evidence for epitope masking because IgG anti-NP administered with NP-SRBC suppressed the IgG anti-NP, but not the IgG anti-SRBC response. Vice versa, IgG anti-SRBC administered with NP-SRBC, suppressed only the IgG anti-SRBC response. In conclusion, passively transferred IgG suppressed all measured parameters of an antigen-specific antibody/B cell response and an important mechanism of action is likely to be epitope masking.

\footnotetext{
Keywords: Fc $\gamma R$, complement, sheep erythrocytes, IgG-mediated immune suppression, rhesus prophylaxis, rhesus $\mathrm{D}$ antigen
}

\footnotetext{
Abbreviations: BCR, B cell receptor; C1q, complement factor 1q; C3, complement factor 3; ELISPOT, enzyme-linked immunospot assay; ELISA, enzyme-linked immunosorbent assay; Fc $\gamma$ R, Fc gamma receptor; FcR $\gamma$, Fc receptor gamma chain; FcRn, the neonatal Fc receptor; GC, germinal center; HEL, hen egg lysozyme; HOD, HEL-OVA-duffy tandem protein; ITIM, immunoreceptor tyrosine-based inhibitory motif; MZ, marginal zone; NP, 4-hydroxy-3-nitrophenyl acetyl; OVA, ovalbumin; PFC, plaque-forming cell assay; RhD, rhesus D antigen; SRBCs, sheep red blood cells; TNP, 2,4,6-trinitrophenyl.
} 


\section{INTRODUCTION}

When antibodies are passively administered together with their specific antigen, they either down- or upregulate the antibody response against this antigen (1-3). IgM, IgG, and IgE enhance antibody responses and their effects depend on interactions of the immune complexes with Fc- or complement receptors, causing more efficient delivery of antigen to splenic B cell follicles and/ or causing enhanced presentation of antigen to $\mathrm{CD} 4^{+} \mathrm{T}$ cells. Interestingly, $\operatorname{IgG}$ has a dual role and the same monoclonal IgG anti-2,4,6-trinitrophenyl that enhances carrier responses when administered with haptenated proteins, suppresses carrier responses when administered with haptenated erythrocytes (4, 5). Since the 1960s, the suppressive capacity of IgG has been used clinically to protect rhesus $\mathrm{D}$ antigen ( $\mathrm{RhD}$ )-negative women from becoming immunized against fetal RhD-positive erythrocytes entering the maternal circulation through transplacental hemorrhage (6). Administration of IgG anti-RhD to these women has significantly reduced the frequency of hemolytic disease of the fetus and newborn (7). The mechanism behind the dramatic ability of IgG to suppress antibody responses has been elusive. It is of substantial theoretical interest to understand how small amounts of $\operatorname{IgG}$ can completely prevent antibody responses to the antigen they recognize. Understanding these mechanisms may also aid in finding effective monoclonal IgG anti-RhD antibodies to use in $\mathrm{RhD}$-prophylaxis.

In the majority of previous studies of IgG-mediated suppression, the number of $\mathrm{B}$ cells producing sheep red blood cell (SRBC)-specific IgM during the first week after immunization was analyzed in mice immunized with native $\mathrm{SRBC} \pm \operatorname{IgG}$ anti-SRBC or haptenated SRBC $\pm \operatorname{IgG}$ anti-hapten. Frequently, more than $99 \%$ of early IgM responses were suppressed (8-13). IgG suppresses also primary IgG responses (14-16) and, when administered immediately prior to a secondary immunization, induction of a secondary antibody response (11). Whether IgG can suppress priming for a memory antibody response against $\mathrm{SRBC}$ is less clear. Some studies demonstrate that IgG-mediated suppression of priming does occur, although it is usually less efficient than suppression of a primary response $(10,14,17,18)$, while others find no suppression (19). IgG does not prevent priming of $\mathrm{T}$ helper cells $(10,15,16)$.

The mechanism underlying IgG-mediated suppression has been intensely investigated over the years. Remarkably, no knockout mouse strain has been found in which suppression does not work. It operates well in mice lacking the activating Fc gamma receptors Fc $\gamma$ RI, Fc $\gamma$ RIII, and FcyRIV (FcR $\gamma$ KO), the inhibitory Fc $\gamma$ RIIB (Fc $\gamma$ RIIB KO), the neonatal Fc receptor $(\mathrm{FcRn})\left(\beta_{2}\right.$-microglobulin $\left.\mathrm{KO}\right)(10,11,20,21)$, as well as complement factor $\mathrm{C} 1 \mathrm{q}(\mathrm{C} 1 \mathrm{q} \mathrm{KO})$, complement factor $\mathrm{C} 3(\mathrm{C} 3 \mathrm{KO})$, or complement receptors 1 and 2 (CR1/2 KO) (21). Moreover, a monoclonal IgG1 antibody, which is unable to activate complement, suppresses to the same degree as a complementactivating IgG1 antibody (22). Therefore, it seems unlikely that IgG-mediated suppression is caused by complement-mediated lysis of SRBC. Because suppression is normal in FcyRIIB KO mice, it is also unlikely that IgG suppresses through central B-cell inhibition, where co-crosslinking of the negatively regulating immunoreceptor tyrosine-based inhibitory motifcontaining FcyRIIB and the B cell receptor (BCR) induces inhibition of $B$ cell signaling (23). Other mechanisms that could explain IgG-mediated suppression are that IgG increases clearance, without involving complement or Fc-receptors, or that IgG masks epitopes on the antigen, and prevents $\mathrm{B}$ cells from binding and becoming activated. Clearance and epitope masking are not mutually exclusive, and mathematical modeling has suggested that both are operative and can act synergistically (24). Passive administration of IgM of different affinities interferes with the development of germinal centers (GCs) and antibody production (25), but whether IgG regulates GC B cells has to our knowledge not been reported previously.

Here, the contribution of clearance and epitope masking has been studied in vivo using passively administered polyclonal SRBC- or 4-hydroxy-3-nitrophenyl acetyl (NP)-specific IgG antibodies as suppressors. The data strongly suggest that epitope masking plays a major role for suppression of IgG responses because only responses against the epitopes recognized by $\operatorname{IgG}$ were suppressed. No correlation was observed between IgGmediated reduction of antigen in the spleen and suppression of the antibody response. In addition, we demonstrate for the first time that IgG suppresses the development of specific extrafollicular antibody-secreting cells, GC B cells as well as long-lived plasma cells. Finally, when primary antibody responses were suppressed to a very high degree ( $>96 \%)$, induction of immunological memory was suppressed to the same extent.

\section{MATERIALS AND METHODS}

\section{Mice}

C57BL/6JBomTac mice (C57BL/6) were from Taconic Bioscience, Inc. (Hudson, NY, USA) and BALB/c mice from Bommice (Ry, Denmark). Fc receptor gamma chain ( $F c R \gamma)$ $\mathrm{KO}$ founders were a gift from Ravetch et al. (26) and were backcrossed to BALB/c for 10 generations. FcR $\gamma$ KO mice lack the common FcR $\gamma$-chain and thereby all activating Fc $\gamma$ Rs associated with this chain (FcyRI, Fc $\gamma$ RIII, and Fc $\gamma$ RIV). Mice were age and sex matched within each experiment and were bred and maintained in the animal facilities of the National Veterinary Institute (Uppsala, Sweden). This study was carried out in accordance with the recommendations of the Uppsala Animal Research Ethics Committee and the protocol was approved by this committee.

\section{Antibodies and Antigens Used for Immunizations}

Polyclonal $\operatorname{IgG}^{a}$ anti-SRBC and polyclonal $\operatorname{IgG}^{a}$ anti-NP were prepared from hyperimmune $\mathrm{BALB} / \mathrm{c}$ serum and polyclonal $\operatorname{IgG}^{\mathrm{b}}$ anti-SRBC from hyperimmune C57BL/6 serum. IgG was purified by affinity chromatography over a Protein-A Sepharose column (Amersham Pharmacia Biotech, Uppsala, Sweden) (27), dialyzed against $\mathrm{PBS}$, sterile filtered and stored at $-20^{\circ} \mathrm{C}$ until use. $\mathrm{IgG}^{\mathrm{a}}$ anti-NP was biotinylated using $0.18 \mathrm{mg}$ EZ-Link Sulfo-NHS-LCLC-Biotin (sulfosuccinimidyl-6-[biotinamido]-6-hexanamido hexaonate) (Thermo Scientific, Waltham, MA, USA) per $2 \mathrm{mg}$ 
IgG according to manufacturer's recommendations. The reaction was performed at room temperature for $30 \mathrm{~min}$ and free biotin was removed by dialysis against PBS. IgG a anti-NP-biotin was sterile filtered and stored at $4^{\circ} \mathrm{C}$ until use. SRBC were acquired from Håtunalab $\mathrm{AB}$ (Håtunaholm, Sweden) and stored in sterile Alsever's solution at $4^{\circ} \mathrm{C}$. SRBC were washed three times in PBS before use. 4-hydroxy-3-nitrophenylacetic-e-aminocaproyl-OSu (NP- $\varepsilon$-Aminocaproyl-OSu) (Biosearch Technologies, Petaluma, CA, USA) was conjugated to SRBC as described before (16). Briefly, NP- $\varepsilon$-Aminocaproyl-OSu was dissolved in dimethylformamide at a concentration of $7.5 \mathrm{mg} / \mathrm{ml}$. Dissolved NP- $\varepsilon$ Aminocaproyl-OSu were then added to 5\% SRBC suspensions in conjugation buffer $\left(0.1 \mathrm{M} \mathrm{NaHCO}_{3}\right.$ with $0.15 \mathrm{M} \mathrm{NaCl}, \mathrm{pH}$ 8.5) to a final concentration of $250 \mu \mathrm{g} / \mathrm{ml}$ (in Figure 3, a final concentration of $1 \mathrm{mg} / \mathrm{ml} \mathrm{NP}-\varepsilon$-Aminocaproyl-OSu was used in order to achieve higher coupling ratio and facilitate visualization in sections) and incubated for $1 \mathrm{~h}$ at room temperature. After the conjugation reaction, cells were washed three times in PBS before use.

\section{Immunization and Blood Sampling}

All mice were immunized with SRBC or NP-SRBC in one of their lateral tail veins in $200 \mu \mathrm{l}$ PBS. Antigen-specific IgG was always administered in $200 \mu \mathrm{l}$ PBS $30 \mathrm{~min}$ prior to antigen, also via the lateral tail veins. Controls received antigen alone or antigen-specific IgG alone. Secondary immunizations were done with SRBC alone. Further details of doses are given in the figure legends. The "default" doses were 30-50 $\mu \mathrm{g}$ IgG and $5 \times 10^{7}$ (NP-)SRBC, both of which are known cause 90-99\% suppression of antibody responses to this amount of erythrocytes. In studies of NP-specific antibody-secreting cells (Figure 3), the higher dose $5 \times 10^{8} \mathrm{NP}-\mathrm{SRBC}$ had to be used to induce detectable numbers of NP-specific cells and the dose of $\operatorname{IgG}$ was correspondingly increased to $100 \mu \mathrm{g}$. In immunological memory experiments, the lower priming dose $5 \times 10^{6}$ SRBC together with $10 \mu \mathrm{g}$ IgG was used (Figure 5A) in addition to the default doses (Figure 5B) to test whether the strength of priming affected whether IgG was able to suppress memory induction or not. Blood was collected from the ventral tail artery.

\section{Enzyme-Linked Immunospot (ELISPOT)}

Enzyme-linked immunospot assay was used for measuring SRBC-specific IgG-secreting cells (28). Briefly, 96-well plates (Costar 96-well enzyme immunoassay/RIA; Sigma-Aldrich, St. Louis, MO, USA) were coated with $0.25 \%$ SRBC. Spleen cells were diluted in cell culture medium (DMEM with $0.5 \%$ FCS), $100 \mu \mathrm{l}$ was added to each well, and the plates were incubated at $37^{\circ} \mathrm{C}$ for $2.5 \mathrm{~h}$. SRBC-specific antibodies produced by the single cells were detected after addition of goat anti-mouse IgGalkaline phosphatase (Jackson ImmunoResearch Laboratories, Inc., West Baltimore Pike, Media, PA, USA) for $3 \mathrm{~h}$ at room temperature, followed by addition of the precipitating substrate 5-bromo-4-chloro-3-indolyl phosphate (Sigma-Aldrich) for $30 \mathrm{~min}$ at room temperature. The plates were washed three times in PBS and samples were counted blindly under a stereomicroscope.

\section{Enzyme-Linked Immunosorbent Assay (ELISA)}

Also NP- and SRBC-specific antibodies in sera were measured by ELISA (16). Briefly, 96-well plates (Costar 96-well enzyme immunoassay/RIA; Sigma-Aldrich) were coated with either $100 \mu \mathrm{l} 0.25 \%$ SRBC or $100 \mu \mathrm{NP}_{20}$-BSA (Biosearch Technologies) $(50 \mu \mathrm{g} / \mathrm{ml})$ in PBS with $0.05 \% \mathrm{NaN}_{3}$. The plates were then blocked with $5 \%$ dry milk at $4^{\circ} \mathrm{C}$ overnight. After washing three times with PBS, serum samples were serially diluted, added to the plates, and incubated overnight at $4^{\circ} \mathrm{C}$. When measuring secondary IgG responses, starting serum dilution for titer determination was 1:5. Cutoff for titers was set as mean $\mathrm{OD}_{405 \mathrm{~nm}}+2 \times \mathrm{SD}$ for a group of eight individual sera from naive BALB/c mice diluted 1:5 (29). When measuring the IgG response, depending on the allotype of injected antibodies, either biotinylated anti-IgG1 ${ }^{\text {b }}$ (clone B68-2) and anti-IgG2 $\mathrm{a}^{\mathrm{b}}$ (clone 5.7) or biotinylated anti-IgG1 ${ }^{\mathrm{a}}$ (clone 10.9) and anti-IgG2a $\mathrm{a}^{\mathrm{a}}$ (clone 8.3) (all from BD Pharmingen, San Jose, CA, USA) were mixed 1:1, added to each well, and incubated overnight at $4^{\circ} \mathrm{C}$. After washing, alkaline phosphatase-conjugated streptavidin (BD Pharmingen, San Jose, CA, USA) was added and incubated for $3 \mathrm{~h}$ at room temperature. After washing, plates were developed using the substrate ( $p$-nitrophenylphosphate; Sigma-Aldrich). Absorbance at $405 \mathrm{~nm}$ was measured and data analyzed using SoftMax software (Molecular Devices, Sunnyvale, CA, USA).

\section{Flow Cytometry}

Spleen cells were prepared as described before (30) and re-suspended in FACS buffer (PBS with 2\% fetal bovine serum). Samples were treated with Fc block (anti-CD16/32; BD Biosciences, San Jose, CA, USA) for 10 min on ice, then stained with antiB220-Alexa700 (clone RA3-6B2), anti-GL7-BV421 (clone GL7), anti-CD95-PEcy7 (clone Jo2), anti- $\lambda 1$-biotin (clone R11-153) (all from $\mathrm{BD}$ Biosciences) at $4^{\circ} \mathrm{C}$ for $30 \mathrm{~min}$. After washing twice in FACS buffer, samples were stained with streptavidin-FITC and $\mathrm{NP}-\mathrm{PE}$ at $4^{\circ} \mathrm{C}$ for $30 \mathrm{~min}$ and re-suspended in $300 \mu \mathrm{l} \mathrm{FACS}$ buffer after washing in FACS buffer. For each sample, 2-3 million events were acquired on a LSR Fortessa cytometer (BD Biosciences) at the BioVis platform, SciLifeLab, Uppsala, Sweden. Data were analyzed with FlowJo software (Tree Star Inc., Ashland, OR, USA).

\section{Confocal Laser Scanning Microscopy}

Spleens were harvested, embedded in optimal cutting temperature embedding compound (VWR international, Radnor, PA, USA), flash-frozen in liquid nitrogen, and stored at $-80^{\circ} \mathrm{C}$. Eightmicrometer sections were cut using Thermo Scientific CryoStar NX70 Cryostat (Thermo Scientific, Waltham, MA, USA), thaw-mounted on frost plus microscope slides (Menzel-Gläser, Braunschweig, Germany), air-dried and stored at $-80^{\circ} \mathrm{C}$ until use. Prior to staining, slides were fixed in $4 \%$ paraformaldehyde (Merck, Darmstadt, Germany) in PBS ( $\mathrm{pH} 7.8$ ) for 15 min or in $50 \%$ acetone for $30 \mathrm{~s}$ followed by $100 \%$ acetone (Sigma-Aldrich) for $5 \mathrm{~min}$. Slides were then rehydrated in PBS and blocked with $5 \%$ horse serum (Sigma-Aldrich) in PBS for $30 \mathrm{~min}$. Slides were stained with primary antibodies for $1 \mathrm{~h}$. After washing twice in PBS, fluorochrome-conjugated streptavidin was added and the 
slides were incubated for $1 \mathrm{~h}$ and washed twice in PBS prior to mounting with Fluoromount $\mathrm{G}$ (Southern Biotech, Birmingham, AL, USA). For detection of NP-specific B cells, slides were stained with anti-IgD-Alexa 488 (clone 11-26C.2a, BioLegend, San Diego, CA, USA) and NP-PE (Biosearch Technologies). Localization of SRBC to splenic marginal zones (MZs) were investigated using anti-B220-Pacific blue (clone RA3-6B2, BD Biosciences), anti-CD169 (MOMA)-FITC (clone MOMA-1, AbD Serotec, Raleigh, NC, USA), and NP-SRBC detection by an in-house produced polyclonal IgG anti-NP-biotin followed by streptavidin-PE (eBioscience, San Diego, CA, USA). Images of immunofluorescence were acquired blindly and randomized with a LSM 700 confocal microscope (Carl Zeiss, Thornwood, NY, USA) using Zen 2009 software (Carl Zeiss). Tile-scan images of whole spleen sections were acquired using Zen 2009 software. Images were analyzed blindly with ImageJ software (NIH, Bethesda, MD, USA).

\section{Statistical Analysis}

Statistical differences between groups were determined by the two-tailed Student's $t$-test. Statistical significance levels were set as: ns, $p>0.05 ;{ }^{*} p<0.05 ;{ }^{* *} p<0.01 ;{ }^{* *} p<0.001$.

\section{RESULTS}

\section{IgG-Mediated Decrease of the Amount of SRBC in the Spleen Is Dependent on Activating Fc $\gamma$ Rs and Does Not Correlate with Suppression}

Specific IgG administered together with SRBC reduces the localization of SRBC in the MZ of the spleen and increases clearance of SRBC from the blood (16). However, after $10 \mathrm{~min}$, the levels of SRBC in the circulation were undetectable, regardless of whether IgG had been co-administered or not (16). The correlation between suppression and antigen localization was not directly assessed nor was the importance of Fc $\gamma$ Rs studied. To investigate this, BALB/c and FcR $\gamma$ KO mice were immunized with IgG antiSRBC $+5 \times 10^{7}$ NP-SRBC, $5 \times 10^{7}$ NP-SRBC alone, or with $1 \times 10^{7} \mathrm{NP}$-SRBC alone. Spleens were harvested after $10 \mathrm{~min}$ and the amount of extracellular NP-SRBC was determined by confocal laser scanning microscopy (Figures 1A-R; enhanced images are shown in Figure S1 in Supplementary Material). In parallel, the serum IgG anti-SRBC response was followed in groups of mice immunized at the same time (Figure 1S). Because the passively administered IgG was obtained from mice with a different IgG allotype than the recipient mice, the actively produced endogenous IgG antibodies could be distinguished by an allotype-specific ELISA (21). Administration of IgG significantly reduced the amount of SRBC in the MZ of wildtype BALB/c mice (Figures 1B,F vs. Figures 1A,E; Figure 1Q) as well as in the entire spleen section (Figure 1R; Figure S2 in Supplementary Material). BALB/c mice immunized with $1 \times 10^{7}$ NP-SRBC alone, or with the fivefold higher amount of NP-SRBC together with IgG anti-SRBC, had comparable amounts of NP-SRBC in their spleens (Figures 1C,G,R). In spite of this, mice immunized with $1 \times 10^{7} \mathrm{NP}-\mathrm{SRBC}$ alone mounted a potent antibody response while mice immunized with IgG anti$\mathrm{SRBC}+5 \times 10^{7} \mathrm{NP}$-SRBC had a suppressed antibody response (Figure 1S). Some spleens were also analyzed for NP-SRBC after $1 \mathrm{~h}$ and $24 \mathrm{~h}$, but, as expected, very little or no antigen could be detected at these time points [Ref. (31) and data not shown]. In FcR $\gamma$ KO mice, IgG was unable reduce the amount of SRBC in the MZ (Figures 1J,N,Q) and in the entire spleen (Figure 1R). In spite of the similar amounts of SRBC in the spleens, the antibody response was suppressed in the IgG group (Figure 1S).

In summary, in $\mathrm{BALB} / \mathrm{c}$ mice, equal (low) levels of antigen in the spleen can result in high $\left(1 \times 10^{7} \mathrm{NP}\right.$-SRBC-group) or suppressed ( IgG $+5 \times 10^{7} \mathrm{NP}$-SRBC-group) antibody responses. In Fc $\gamma \mathrm{R}$ KO mice, equal (high) levels of antigen can result in high $\left(5 \times 10^{7} \mathrm{NP}\right.$-SRBC) or suppressed (IgG $+5 \times 10^{7} \mathrm{NP}$-SRBC-group) antibody responses. This lack of correlation between suppression and the amount of NP-SRBC detected in the spleen is hard to reconcile with clearance of antigen as the major explanation for IgG-mediated suppression. Moreover, the observations confirm that administration of SRBC-specific IgG reduces the amount of antigen localized in the spleen (16) and demonstrate that the reduction is dependent on activating Fc $\gamma$ Rs while the suppression of antibody responses is not.

\section{Epitope-Specific Suppression of IgG Responses}

Next, we sought to determine whether IgG only suppresses responses against the epitopes to which it binds (epitope-specific suppression) or whether it, in addition, suppresses responses against other epitopes on the same antigen (non-epitope specific suppression). To this end, C57BL/6 mice were immunized with NP-SRBC alone or together with either IgG anti-NP or IgG anti-SRBC. The mice were bled every 2 weeks and their antibody responses against NP and SRBC were analyzed. IgG anti-NP suppressed the NP- but not the SRBC-specific IgG-responses (Figures 2A,B) while IgG anti-SRBC suppressed the SRBC- but not the NP-specific IgG response (Figures 2C,D). These observations were highly reproducible (4/4 experiments with IgG anti$\mathrm{NP}$ and $2 / 2$ with IgG anti-SRBC) and demonstrate that IgG is able to suppress IgG responses in an epitope-specific way.

\section{IgG Suppresses NP-Specific Extrafollicular Antibody-Secreting Cells and NP-Specific GC B Cells}

In previous studies, the ability of IgG to suppress the overall serum antibody levels or splenic antibody-secreting cells was analyzed. Here, we investigated which B cell subpopulations were suppressed by IgG, using a system in which the BCRs of the antigen-specific B cells could be stained directly. The antibody response against $\mathrm{NP}$ in $\mathrm{C} 57 \mathrm{BL} / 6$ mice is genetically restricted, mainly comprising $\lambda 1$ light chains and the V186.2 segment of the VHJ558 gene family (32). Therefore, staining for $\lambda 1$ and NP can be used to identify NP-specific B cells. C57BL/6 mice were immunized with NP-SRBC \pm IgG anti-NP and negative controls with unconjugated SRBC or left unimmunized. Six days later, spleens were analyzed for NP-binding cells with flow cytometry and confocal microscopy (Figure 3). In mice immunized with 


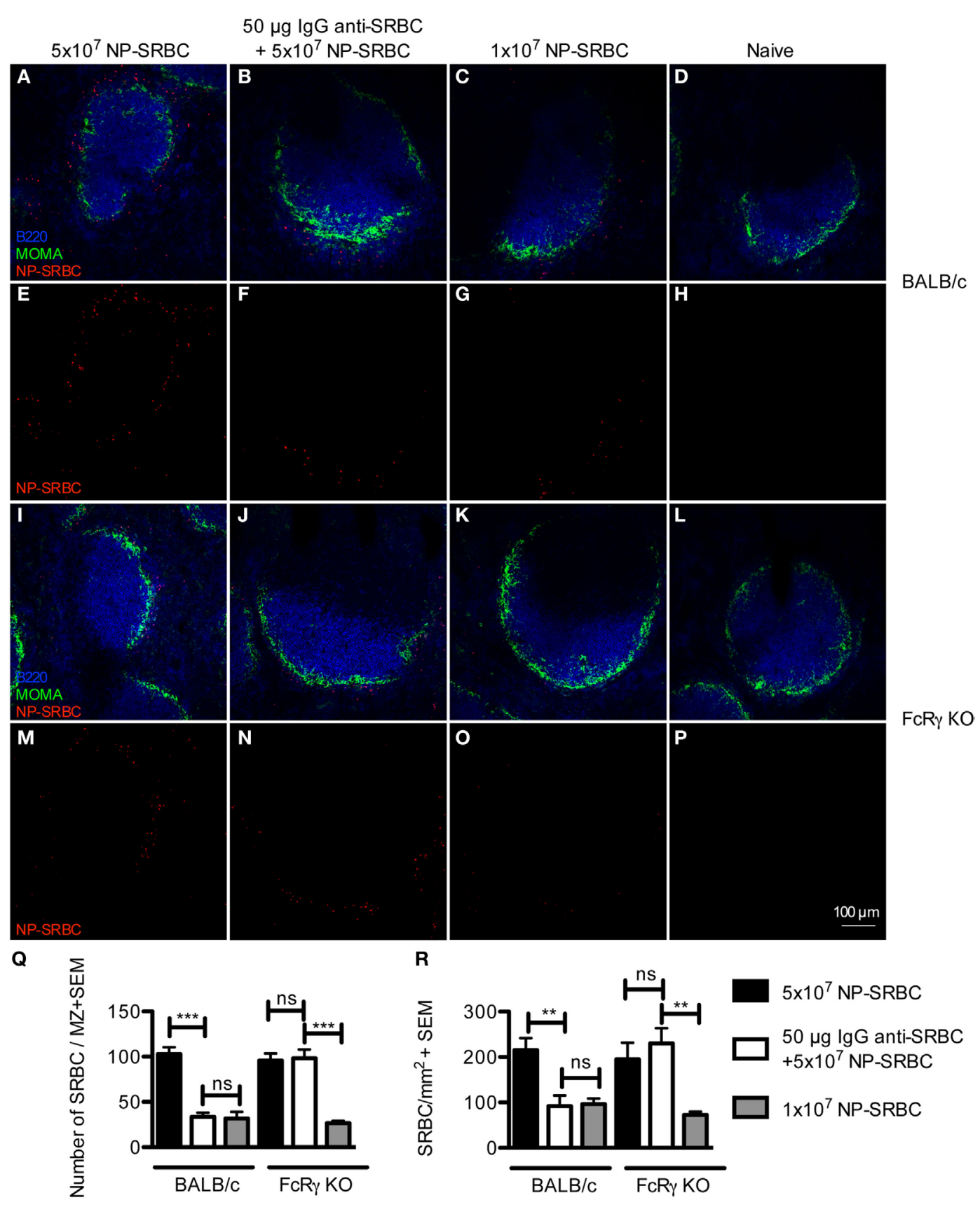

S

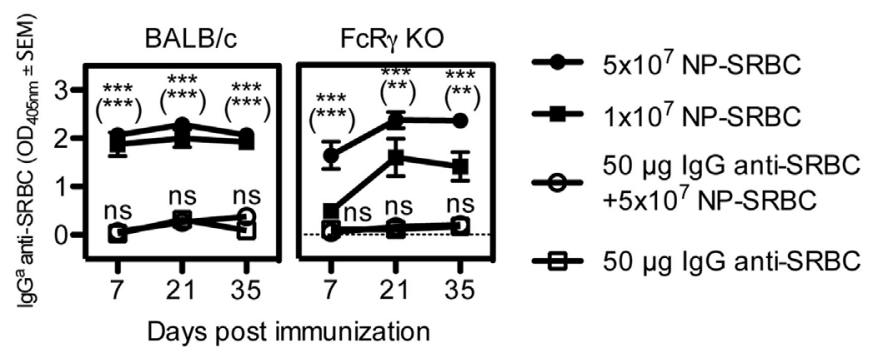


FIGURE 1 | Continued

IgG-mediated decrease of the amount of sheep red blood cell (SRBC) in the spleen is dependent on activating Fc $\gamma$ Rs and does not correlate with suppression. BALB/C and FCR $\mathrm{KO}$ mice were immunized with $5 \times 10^{7} \mathrm{NP}-\mathrm{SRBC} \pm 50 \mu \mathrm{g}$ lgG ${ }^{\mathrm{b}}$ anti-SRBC or $1 \times 10^{7} \mathrm{NP}_{-} \mathrm{SRBC}$. Naive mice and mice-receiving IgG ${ }^{b}$ anti-SRBC alone were used as negative controls. Spleens were harvested 10 min after administration of NP-SRBC and processed for analysis by confocal laser scanning microscopy ( $n=5$ /group). (A-P) Visualization of NP-SRBC localization in spleen sections: B220+ B cells (blue), MOMA+ metallophilic macrophages (green), and NP-SRBC (red). Imaging of samples was performed blindly. (Q) Quantification of the number of NP-SRBC per marginal zone. Ten to fifteen randomly selected follicles per spleen per mouse were imaged at 20x magnification. (R) Quantification of the number of NP-SRBC per millimeter square in whole spleen sections. Tile scans of one whole spleen section per mouse were imaged at 10x magnification. (S) The IgG anti-SRBC response was followed in parallel in mice from each group for 7-35 days after immunization ( $n=5$ /group, $n=3$ for negative controls). Sera diluted 1:125 were screened for lgG a anti-SRBC in enzyme-linked immunosorbent assay. $p$-Values for comparisons of mice immunized with IgG anti-SRBC and NP-SRBC vs $5 \times 10^{7}$ NP-SRBC alone are given without parentheses. Comparisons of mice immunized with IgG anti-SRBC and $5 \times 10^{7} \mathrm{NP}-\mathrm{SRBC}$ vs $1 \times 10^{7} \mathrm{NP}-\mathrm{SRBC}$ alone are given within parentheses. Data are representative of two (S: BALB/c), one (S: FCR $\mathrm{KO}$ ), or at least three experiments (A-R). ns, $p>0.05 ;{ }^{*} p<0.05 ;{ }^{* \star} p<0.01 ;{ }^{* \star *} p<0.001$.

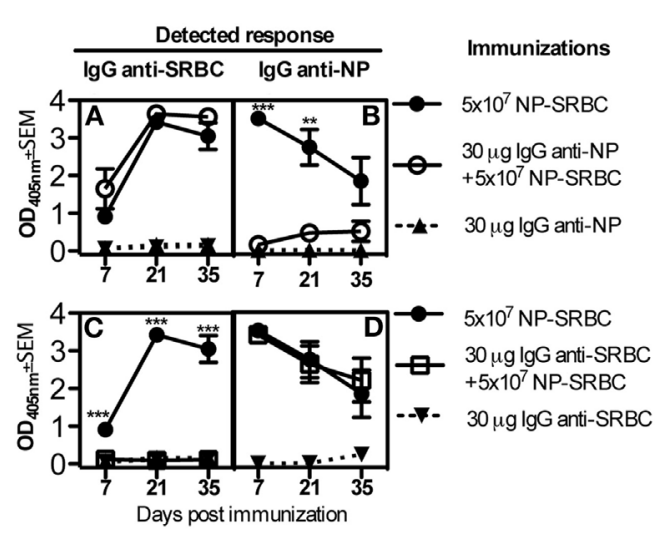

FIGURE 2 | Epitope-specific suppression of IgG responses. C57BL/6 mice were immunized with $5 \times 10^{7} \mathrm{NP}$-sheep red blood cells (SRBC) $\pm 30 \mu \mathrm{g}$ $\operatorname{lgG}^{\mathrm{a}}$ anti-NP or $(\mathbf{A}, \mathbf{B})$ with $5 \times 10^{7} \mathrm{NP}-\mathrm{SRBC} \pm 30 \mu \mathrm{g} \lg \mathrm{G}^{\mathrm{a}}$ anti-SRBC (C,D). IgG was also administered alone as negative control. Serum samples were diluted 1:125 and analyzed in enzyme-linked immunosorbent assay. Representative of four (A,B) or two (C,D) experiments; $n=5$ mice/group (except for lgG alone where $n=2$ mice/group). Statistical comparisons were done between solid circles vs. open circles in (A,B) and between solid circles vs. open squares in (C,D). ns, $p>0.05 ;{ }^{\star} p<0.05 ;{ }^{\star \star} p<0.01 ;{ }^{\star \star *} p<0.001$.

NP-SRBC alone, a small population of $\lambda 1^{+} \mathrm{NP}^{+} \mathrm{B} 220^{+}$cells were identified both among the GL7 ${ }^{\text {high }}$ CD95 $5^{\text {high }}$ GC B cells (Figure 3B bottom right panel; Figure 3C) and among the GL7 ${ }^{\text {low }}$ CD95 $5^{\text {low }}$ non-GC B cells (Figure 3B bottom left panel; Figure 3C). The NP-specific cells (GC B cells and non-GC $\mathrm{B}$ cells together) constituted approximately $0.05 \%$ of the total $\mathrm{B} 220^{+}$population and $\sim 1 / 5$ were GC B cells and $\sim 4 / 5$ non-GC $B$ cells (Figure 3C). Administration of IgG anti-NP together with NP-SRBC abolished the induction of NP-specific GC and non-GC B cells (Figures 3A,C). In fact, the levels in these mice were equally low as in mice immunized with unconjugated SRBC or left untreated (Figure 3C).

The other half of the spleens were analyzed in confocal microscopy (Figures 3D-I). NP-binding cells were readily detected in extrafollicular foci in mice immunized with NP-SRBC (Figure 3E) while very few NP-binding cells were detected in mice immunized with IgG anti-NP + NP-SRBC (Figure 3D) and in the negative controls (Figures 3F,G). Although it cannot be excluded that some of the NP-binding cells in extrafollicular foci had exited GCs, the majority are presumably true extrafollicular cells owing to the early time point at which they were analyzed. Importantly, all NP-specific cells outside the follicles, regardless of their origin, are absent in mice immunized with IgG anti-NP + NP-SRBC. NP-specific cells could also be detected in some GCs (Figure 3H). GC B cells stained less brightly than the extrafollicular NP-specific cells possibly due to a wider span in affinity (33) and lower Ig expression. To confirm that the unstained areas close to the $\mathrm{IgD}^{+}$areas are indeed GCs, costaining with PNA was performed (Figure S3 in Supplementary Material). In summary, IgG anti-NP can suppress the generation of both extrafollicular NP-specific antibody-secreting cells and NP-specific GC B cells.

\section{IgG Suppresses the Generation of Long-Lived Plasma Cells}

To determine whether IgG suppressed the development of long-lived plasma cells, mice were immunized with SRBC \pm IgG anti-SRBC and the number of SRBC-specific IgG-secreting cells in the spleen and bone marrow was analyzed 5-70 days after immunization (Figure 4). Cells secreting IgG anti-SRBC were detected in both organs at all times, but were severely suppressed in the groups receiving IgG together with SRBC. As expected, the number of antibody-producing cells in the spleen was highest early after immunization and then decreased, while the cell number in the bone marrow increased during the entire time period. Thus, administration of IgG anti-SRBC efficiently suppressed the specific IgG-secreting cells at all times, both in spleen and bone marrow.

\section{IgG Suppresses Induction of Immunological Memory}

To test the ability of IgG to regulate memory induction, BALB/c mice were immunized in two regimes: $5 \times 10^{7} \mathrm{SRBC} \pm 50 \mu \mathrm{g}$ IgG anti-SRBC or $5 \times 10^{6} \mathrm{SRBC} \pm 10 \mu \mathrm{g}$ IgG anti-SRBC. The IgG anti-SRBC responses were followed during 63 days after priming, confirming efficient suppression (Figures 5A,B). And, 70 days after priming, all mice were boosted with a suboptimal number of SRBC and, in addition, a group of naive mice received the same "booster" dose. Because of the great differences in antibody levels between the groups, we analyzed the antibody responses as endpoint titers, starting at a serum dilution of 1:5. In mice that had been primed with SRBC alone 
and boosted, a very high secondary antibody response was observed (Figures 5A,B). In mice primed with IgG + SRBC and boosted, secondary responses were low (Figures 5A,B). A minute priming effect was, however, visible also in the IgG groups, evidenced by the fact that their antibody titers were higher than those in naive and "boosted" mice (Figures 5A,B). Naive mice, primed on day 70 with the suboptimal dose $5 \times 10^{5}$ SRBC, produce low titers of IgG antibodies [mean titers: 30 (Figure 5A) and 1,100 (Figure 5B)], which are barely visible in the figures. The relative suppression of primary responses and induction of memory appeared to be equal. In the low dose experiment, IgG suppressed $96 \%$ of the IgG-response on day 21 and $99 \%$ on day 91 (21 days after boost) (Figure 5A). In the high dose experiment, suppression was $97 \%$ both 21 days after priming and 21 days after boost (Figure 5B). Thus, when IgG-mediated suppression of primary antibody responses is very efficient, priming for secondary antibody responses seems to follow along the same lines.

\section{DISCUSSION}

Generation of an adaptive antibody response is a complicated process, and one purpose of the present investigation was to define in detail which parameters along this line that can be suppressed by IgG. After the initial interaction between antigen, $\mathrm{B}$ cells, and cognate $\mathrm{T}$ cells, the specific $\mathrm{B}$ cells can differentiate into extrafollicular short-lived IgM- or IgG-secreting cells, or GC-independent memory B cells. Other B cells enter the GC pathway, go through hypermutation followed by selection for high affinity and class switch recombination becoming either GC-dependent memory B cells or long-lived plasma cells [reviewed in Ref. (34-36)]. Differentiation between IgG-mediated suppression of extrafollicular B cells, non-GC B cells, and GC $B$ cells in the spleen is not possible by just looking at serum antibodies or direct plaque-forming cell assay. In order to detect single antigen-specific B cells in vivo, usually Ig-transgenic mice immunized with antigen in adjuvants are used. Here, we managed

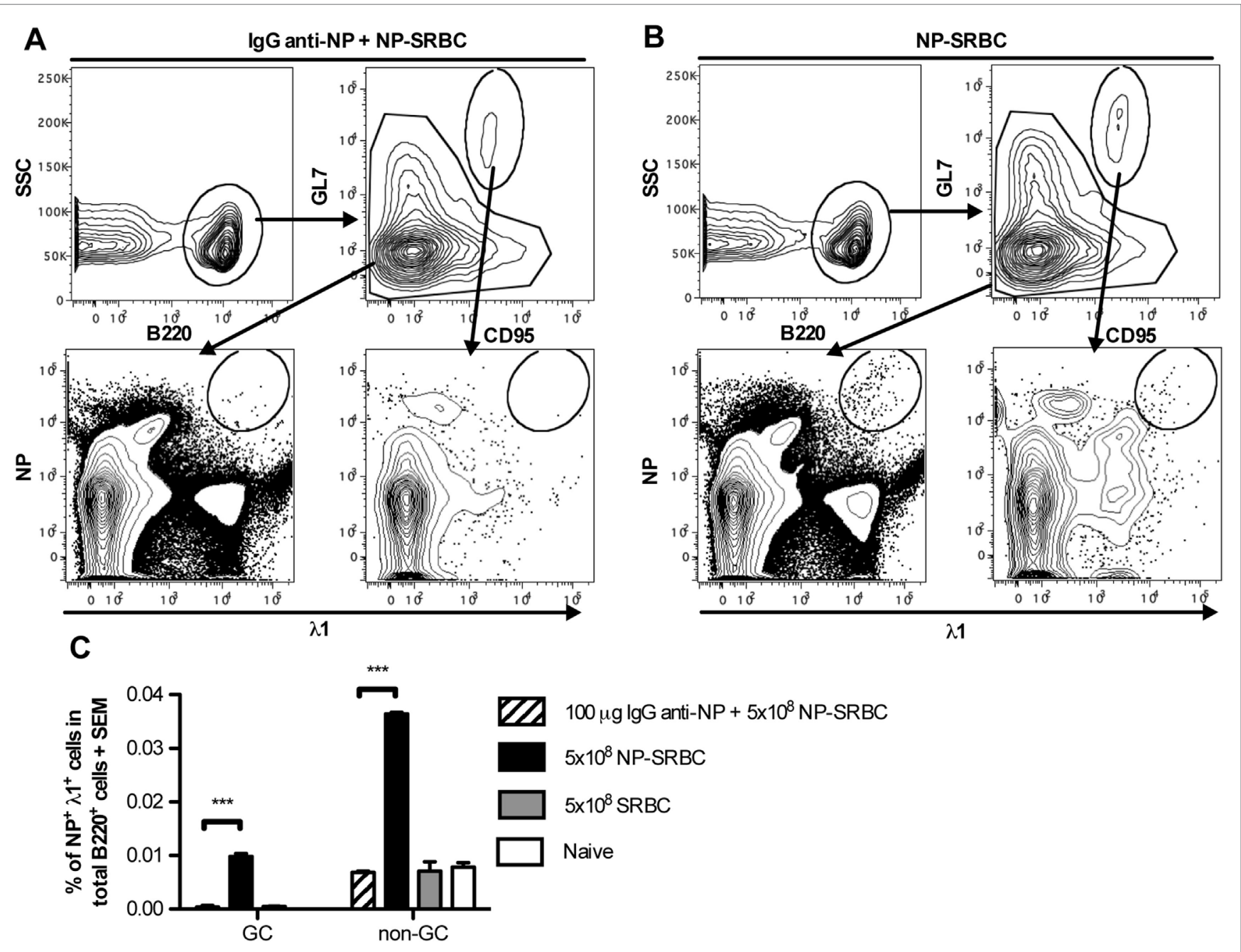

FIGURE 3 | Continued 

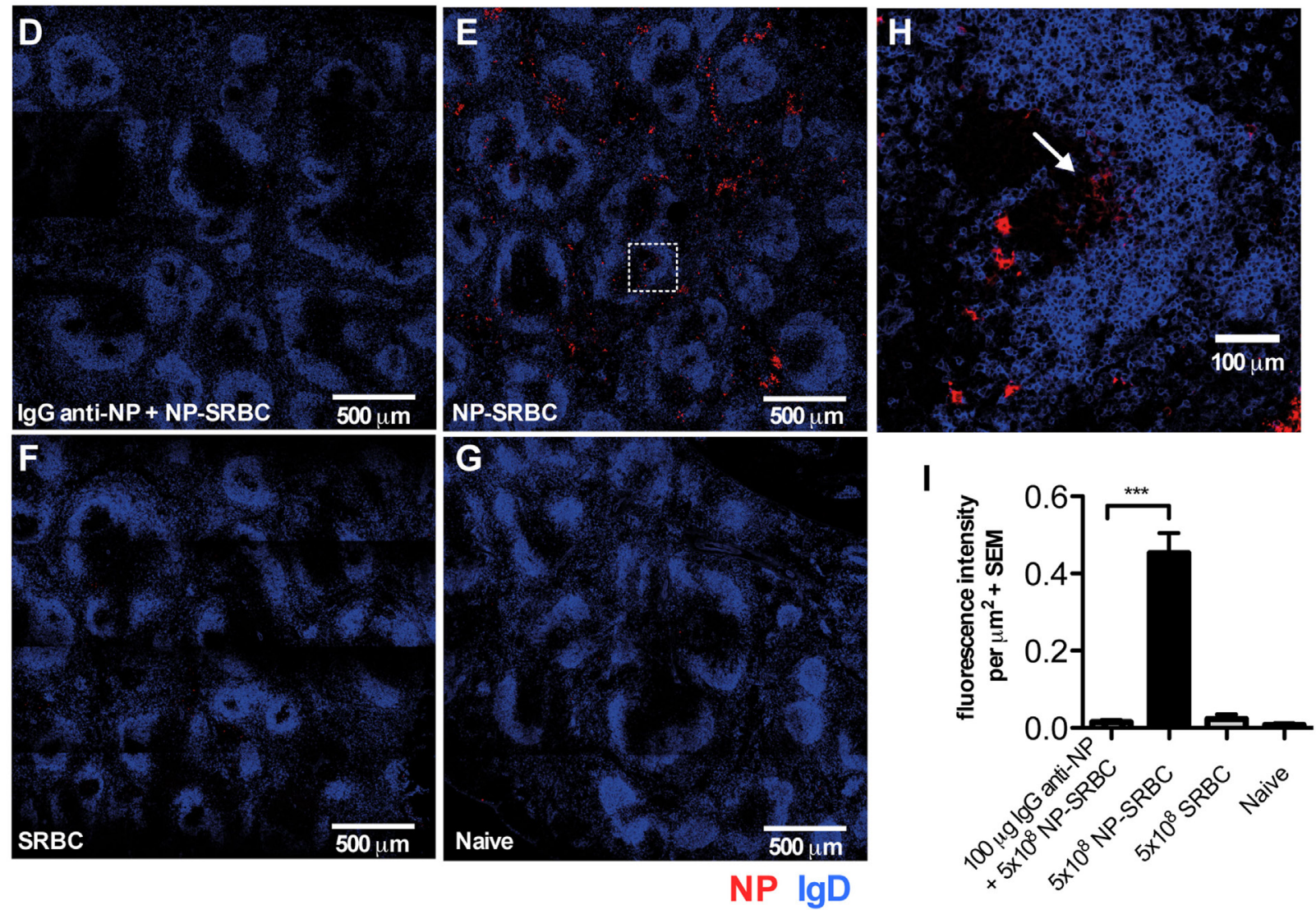

FIGURE 3 | IgG suppresses NP-specific extrafollicular antibody secreting cells and NP-specific germinal centers (GCs) B cells. C57BL/6 mice were immunized with $5 \times 10^{8} \mathrm{NP}$-sheep red blood cell (SRBC) $\pm 100 \mu \mathrm{g}$ polyclonal lgG ${ }^{\mathrm{a}}$ anti-NP, $5 \times 10^{8}$ unconjugated SRBC, or left untreated. After 6 days, spleens were harvested and half of the spleen samples were analyzed with flow cytometry (A-C) and the other half with confocal microscopy (D-I). (A,B) Gating strategy for GC and non-GC NP+ $\lambda 1^{+}$cells. (C) Frequency of GC and non-GC NP+ $\lambda 1^{+}$cells among total B220 ${ }^{+}$cells. (D-G) Representative tile scan sections, stained for lgD+ (blue), and $\mathrm{NP}^{+}$(red) cells. (H) Enlarged image from (E) (square with dashed lines). (I) Quantification of fluorescence intensity per square micrometer. One representative tile scan image was taken from each mouse and the intensity of red fluorescence ( $\mathrm{NP}^{+}$) from each image was summed and then divided by the size of the image. Representative of three independent experiments with $2-4$ mice/group. ns, $p>0.05 ;{ }^{*} p<0.05 ;{ }^{\star \star} p<0.01 ;{ }^{\star \star \star} p<0.001$.

to set up a system in which i.v. immunization of C57BL/6 mice with $5 \times 10^{8} \mathrm{NP}$-SRBC in PBS resulted in NP-specific B cell populations, which were detectable through staining of specific BCR with NP-PE. Administration of IgG anti-NP completely prevented development of NP-specific extrafollicular B cells, non-GC B cells, and GC B cells (Figure 3). IgG anti-SRBC also suppressed the development of long-lived plasma cells in bone marrow obtained up to 70 days after immunization (Figure 4).

An important function of the adaptive immune system is the generation of long-lived memory B cells. Whether IgG, acting to suppress a primary response, also inhibits priming for immunological memory has not been unequivocally determined $(10,14$, 17-19). By using an ELISA that discriminates between passively administered and actively produced, endogenous IgG, and by measuring the IgG levels in the serum as endpoint titers, we could directly compare the relative suppression of primary and secondary IgG responses. The data show that when primary responses were suppressed by $96 \%$ or more, secondary responses were also suppressed by $96 \%$ or more. Thus, the relative suppression of a primary IgG response and priming for a secondary IgG response is very similar (Figure 5). This is in accordance with current knowledge about the development of memory B cells, most of which are generated during the first weeks of an immunization (36). Therefore, it would be expected that lack of B cell stimulation, owing to IgG-mediated inhibition of the interaction between antigen and B cells, during this time, would result in lack of memory B cells. When the primary B cell response is only partially suppressed, a partial suppression of priming would be the logical result, and this may explain why priming has sometimes been found to be only moderately suppressed. The observation is also consistent with the successful $\mathrm{RhD}$ prophylaxis in humans in which administration of $\operatorname{IgG}$ anti-RhD to women at the first pregnancy with a $\mathrm{RhD}$-positive baby protects against immunization during the next pregnancy $(6,7)$.

To further elucidate the mechanism underlying IgG-mediated suppression, we focused on differentiating between the two hypotheses that are currently mainly discussed, antigen clearance and epitope masking. Provided Fc $\gamma$ Rs were expressed in the mice, 
IgG was able to increase clearance, but no correlation between the antibody response and the amount of NP-SRBC found in the spleens was observed (Figure 1). These findings are consistent with previous observations. IgG administered several days after SRBC causes suppression $(11,12,37-40)$ in spite of the fact that SRBC is cleared from the circulation of mice within $10 \mathrm{~min}$ of

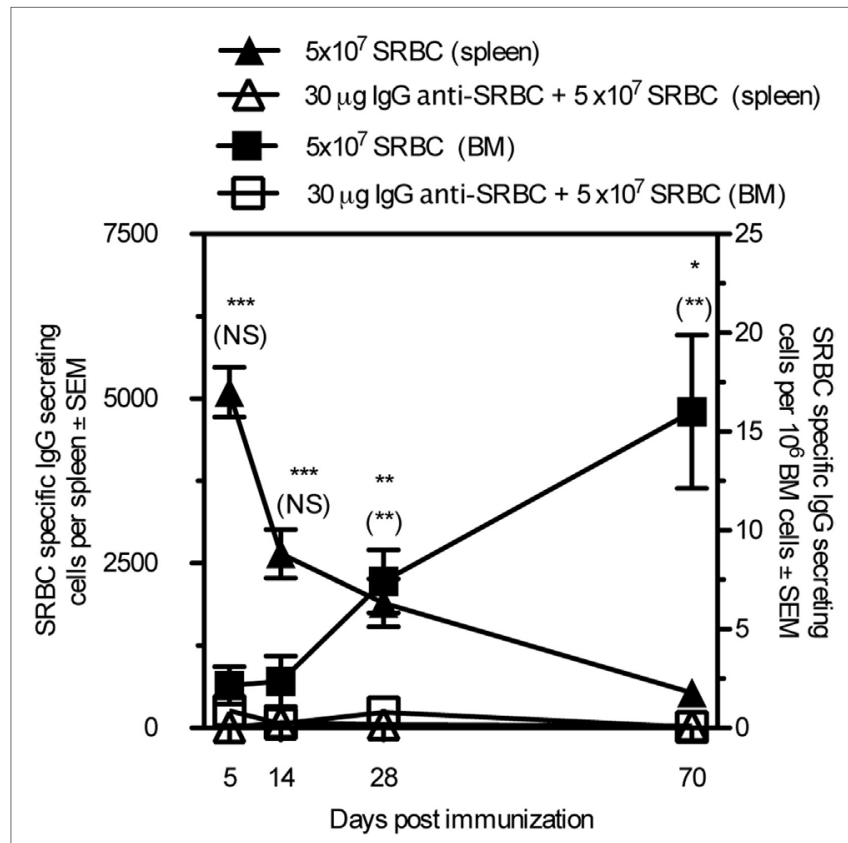

FIGURE 4 | IgG suppresses the generation of long-lived plasma cells. BALB/c mice were immunized with $5 \times 10^{7}$ sheep red blood cell $(\mathrm{SRBC}) \pm 30 \mu \mathrm{g} \lg \mathrm{G}^{\mathrm{b}}$ anti-SRBC. Spleens and bone marrow were harvested and SRBC-specific antibody-secreting cells were measured with ELISPOT.

Comparisons between filled and open triangles are given without parentheses and comparisons between filled and open squares are given within parentheses. ns, $p>0.05 ;{ }^{*} p<0.05 ;{ }^{* *} p<0.01$; ${ }^{* \star *} p<0.001$. immunization (16). In a model using as antigen transgenic mouse erythrocytes (HOD-RBC), which express hen egg lysozyme in tandem sequence with $\mathrm{T}$ cell determinants of ovalbumin (OVA) and the complete human Duffy ${ }^{\mathrm{b}}$ transmembrane protein (41), a panel of monoclonal IgG antibodies were tested for ability to suppress the OVA-specific antibody response. Three of the antibodies induced clearance while three did not, but all six were efficient suppressors (42). In addition, monoclonal anti-RhD administered together with $\mathrm{RhD}^{+}$erythrocytes to $\mathrm{RhD}^{-}$subjects induced rapid clearance of erythrocytes but failed to suppress the antibody response $(43,44)$.

Direct evidence for a role of epitope masking in IgG-mediated suppression is difficult to obtain, and this hypothesis will probably remain a "diagnosis by exclusion." Epitope masking by IgM antibodies was, however, suggested to play an important role in selection of B cells in germinal centers (25), and to be the most important explanation for poor secondary antibody responses to the stem of the influenza surface hemagglutinin during vaccinations (45). The highly reproducible epitope specificity of suppression of the IgG responses shown in Figure 2 lends strong support for the epitope masking hypothesis. This finding may be the closest to a direct proof for the epitope masking hypothesis, which is possible to obtain. Importantly, epitope specificity of suppression has been reported previously $(12,46)$. However, also non-epitope specific suppression has been observed, mainly in systems studying IgM-producing single B cells during the first week of immunization $(4,9,10,13,16,22,42)$. Because non-epitope specificity has been interpreted as evidence for Fc-dependence of suppression and, therefore, to argue against the epitope masking mechanism, this is an important issue. The existence of non-epitope specific suppression needs to be accommodated with the increasing support for Fc-independence of suppression obtained from studies of Fc $\gamma \mathrm{R}$ - and complement-deficient mice $(10,11,20-22)$ and the observations that $\mathrm{F}\left(\mathrm{ab}^{\prime}\right)_{2}$ fragments can suppress $(10,20,47,48)$. We have suggested $(10,49)$, and still believe, that the best explanation for the apparent paradox of lack

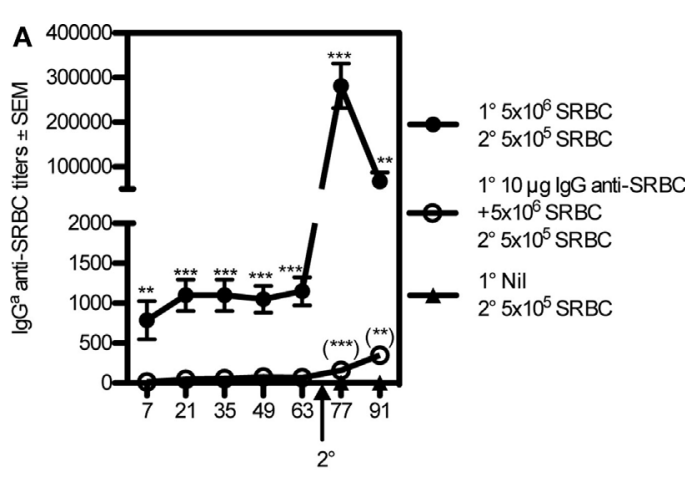

Days post $1^{\circ}$ immunization

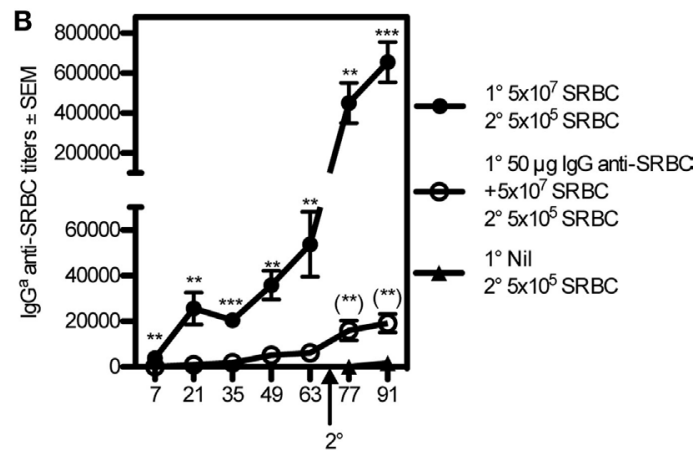

Days post $1^{\circ}$ immunization

FIGURE 5 | IgG suppresses the induction of immunological memory. BALB/c mice were immunized with $5 \times 10^{6}$ sheep red blood cell (SRBC) $\pm 10 \mu g$ lgG anti-SRBC+ or (A) or $5 \times 10^{7} \mathrm{SRBC} \pm 50 \mu \mathrm{g} \mathrm{lgG}$ anti-SRBC (B). And, 70 days after priming, all mice were boosted with $5 \times 10^{5}$ sheep red blood cells (SRBCs). An additional, unprimed, group also received $5 \times 10^{5} \mathrm{SRBC}$ at this time point (filled triangles). Serum IgG anti-SRBC titers were assayed in enzyme-linked immunosorbent assay. $p$-Values for comparisons between mice primed with lgG anti-SRBC and $5 \times 10^{7} \mathrm{SRBC}$ vs. $5 \times 10^{7} \mathrm{SRBC}$ alone are given without parentheses. Comparisons between mice primed with IgG anti-SRBC and SRBC and boosted with $5 \times 10^{5}$ SRBC vs. naive mice "boosted" with $5 \times 10^{5}$ SRBC alone are given within parentheses. Data are representative of at least three experiments per antigen dose $\left(n=5-8 /\right.$ group). ns, $p>0.05 ;{ }^{* \star} p<0.01$; ${ }^{\star \star \star} p<0.001$. 
of Fc-dependence and existence of non-epitope specific suppression is steric hindrance. IgG binding to an epitope present at high density prevents B cells from binding to the specific epitope (via epitope masking) as well as to neighboring non-specific epitopes (via steric hindrance). When IgG binds to an epitope present at low density, it would only prevent B cells from binding to the specific epitope. Thus, in conditions with high epitope density, non-epitope specific suppression will be observed and in conditions with low epitope density, epitope-specific suppression will take place. With this reasoning, both epitope-specific and non-epitope-specific suppression can be understood, although suppression is independent of the $\operatorname{IgG}(\mathrm{Fc})$ part. Experimental support for this idea comes from studies where hapten-specific IgG antibodies suppressed non-epitope-specific SRBC-responses when administered together with high hapten-density SRBC, but not with low hapten-density $\operatorname{SRBC}(5,16)$. In analogy, the amount of IgG that binds to SRBC correlates with how efficiently suppression is induced $(9,50)$, mixtures of passively administered different monoclonal IgG antibodies induce more efficient suppression than administration of saturating doses of each IgG antibody alone $(9,51,52)$, and high affinity of IgG promotes efficient suppression $(13,53)$. In humans, a strong argument against the epitope masking mechanism has been a study in which anti-Kell antibodies could suppress responses against $\mathrm{RhD}$ epitopes (54). However, it was recently shown that the $\mathrm{RhD}$ and Kell antigens are positioned closely together on human erythrocytes (55) and, therefore, these observations may perhaps be explained by epitope masking in combination with steric hindrance.

Compatible with epitope masking is also the lack of correlation between clearance and suppression, discussed above, and the fact that $\mathrm{T}$ cells are not suppressed by $\operatorname{IgG}(10,15,16)$. This is what would be expected because epitope masking would not prevent antigen from being internalized and processed by the APCs, which subsequently activate $\mathrm{CD}^{+} \mathrm{T}$ cells. Obviously, some experimental data are not compatible with the epitope masking hypothesis. For example, $\mathrm{F}\left(\mathrm{ab}^{\prime}\right)_{2}$ fragments have been reported to be unable to suppress $(4,13,17)$. This can possibly be explained by increased elimination of these fragments owing to lack of binding to FcRn (56), but this issue needs further experimentation. Another argument against epitope masking comes from the HOD-RBC model, in which a monoclonal IgG anti-Duffy antibody was able to non-epitope specifically suppress anti-HEL responses although it did not sterically block binding

\section{REFERENCES}

1. Heyman B. Regulation of antibody responses via antibodies, complement, and Fc receptors. Annu Rev Immunol (2000) 18:709-37. doi:10.1146/annurev. immunol.18.1.709

2. Heyman B. Antibodies as natural adjuvants. Curr Top Microbiol Immunol (2014) 382:201-19. doi:10.1007/978-3-319-07911-0_9

3. Nimmerjahn F, Ravetch JV. Antibody-mediated modulation of immune responses. Immunol Rev (2010) 236:265-75. doi:10.1111/j.1600-065X.2010. 00910.x

4. Enriquez-Rincon F, Klaus GG. Differing effects of monoclonal anti-hapten antibodies on humoral responses to soluble or particulate antigens. Immunology (1984) 52:129-36.

5. Wiersma EJ, Coulie PG, Heyman B. Dual immunoregulatory effects of monoclonal IgG-antibodies: suppression and enhancement of the antibody of serum IgM anti-HEL to HOD-RBC (42). However, it remains to be elucidated how well competition with serum IgM resembles the in vivo situation where B cells themselves compete with the suppressive IgG.

In addition to the use of suppressive IgG in $\mathrm{RhD}$ prophylaxis, an important clinical issue is the negative impact of passively transferred pathogen-specific maternal antibodies on vaccination of infants [reviewed in Ref. (57-59)]. Also within this field, the mechanisms discussed are Fc $\gamma$ RIIB-mediated inhibition, clearance, and epitope masking, but so far, no concensus has been reached.

In summary, the current study extends our understanding of IgG-mediated suppression in two principal ways. First, it suggests that IgG administered in close temporal relationship to antigen inhibits all pathways of B cell differentiation. Serum IgG as well as extrafollicular B cells, non-GC B cells, GC B cells, long-lived plasma cells, and memory B cells are all suppressed. Second, it provides strong evidence for the importance of epitope masking and suggests that the role of IgG-mediated antigen clearance is negligible.

\section{AUTHOR CONTRIBUTIONS}

JB and HX: designed and performed experiments, and wrote the manuscript. $\mathrm{BH}$ : designed experiments and wrote the manuscript.

\section{ACKNOWLEDGMENTS}

We thank Annika Westin for skillful technical assistance. This work was supported by Uppsala University, The Swedish Research Council, China Scholarship Council, Ellen, Walter, and Lennart Hesselman's Foundation, Hans von Kantzow's Foundation, King Gustaf V:s 80 Years Foundation, Ollie and Elof Ericsson's Foundation, and Agnes and Mac Rudberg's Foundation. The flow cytometric analyses were performed on instruments provided by the SciLifeLab BioVis platform at Uppsala University.

\section{SUPPLEMENTARY MATERIAL}

The Supplementary Material for this article can be found online at http://journal.frontiersin.org/article/10.3389/fimmu.2017.00238/ full\#supplementary-material.

response. Scand J Immunol (1989) 29:439-48. doi:10.1111/j.1365-3083.1989. tb01143.x

6. Clarke CA, Donohoe WTA, Woodrow JC, Finn R, Krevans JR, Kulke W, et al. Further experimental studies on the prevention of $\mathrm{Rh}$ haemolytic disease. Br Med J (1963) 1:979-84. doi:10.1136/bmj.1.5336.979

7. Urbaniak SJ, Greiss MA. RhD haemolytic disease of the fetus and the newborn. Blood Rev (2000) 14:44-61. doi:10.1054/blre.1999.0123

8. Henry C, Jerne N. Competition of $19 \mathrm{~S}$ and $7 \mathrm{~S}$ antigen receptors in the regulation of the primary immune response. J Exp Med (1968) 128:133-52. doi:10.1084/jem.128.1.133

9. Heyman B, Wigzell H. Immunoregulation by monoclonal sheep erythrocyte-specific IgG antibodies: suppression is correlated to level of antigen binding and not to isotype. J Immunol (1984) 132:1136-43.

10. Karlsson MC, Wernersson S, Diaz de Stahl T, Gustavsson S, Heyman B. Efficient IgG-mediated suppression of primary antibody responses in 
Fcgamma receptor-deficient mice. Proc Natl Acad Sci U S A (1999) 96:2244-9. doi:10.1073/pnas.96.5.2244

11. Karlsson MC, Getahun A, Heyman B. FcgammaRIIB in IgG-mediated suppression of antibody responses: different impact in vivo and in vitro. J Immunol (2001) 167:5558-64. doi:10.4049/jimmunol.167.10.5558

12. Möller G. Antibody-mediated suppression of the immune response is determinant specific. Eur J Immunol (1985) 15:409-12. doi:10.1002/eji. 1830150420

13. Brüggemann M, Rajewsky K. Regulation of the antibody response against hapten-coupled erythrocytes by monoclonal anti-hapten antibodies of various isotypes. Cell Immunol (1982) 71:365-73. doi:10.1016/0008-8749(82) 90270-2

14. Heyman B, Wigzell H. Specific IgM enhances and IgG inhibits the induction of immunological memory in mice. Scand J Immunol (1985) 21:255-66. doi:10.1111/j.1365-3083.1985.tb01428.x

15. Brinc D, Le-Tien H, Crow AR, Freedman J, Lazarus AH. IgG-mediated immunosuppression is not dependent on erythrocyte clearance or immunological evasion: implications for the mechanism of action of anti-D in the prevention of haemolytic disease of the newborn? Br J Haematol (2007) 139:275-9. doi:10.1111/j.1365-2141.2007.06764.x

16. Getahun A, Heyman B. Studies on the mechanism by which antigen-specific IgG suppresses primary antibody responses: evidence for epitope masking and decreased localization of antigen in the spleen. Scand J Immunol (2009) 70:277-87. doi:10.1111/j.1365-3083.2009.02298.x

17. Nicholas R, Sinclair SC. Regulation of the immune response. I. Reduction in ability of specific antibody to inhibit longlasting IgG immunological priming after removal of the Fc fragment. J Exp Med (1969) 129:1183-201. doi:10.1084/jem.129.6.1183

18. Safford JW Jr, Tokuda S. Antibody-mediated suppression of the immune response: effect on the development of immunologic memory. J Immunol (1971) 107:1213-25.

19. Brinc D, Le-Tien H, Crow AR, Siragam V, Freedman J, Lazarus AH. Immunoglobulin G-mediated regulation of the murine immune response to transfused red blood cells occurs in the absence of active immune suppression: implications for the mechanism of action of anti-D in the prevention of haemolytic disease of the fetus and newborn? Immunology (2008) 124:141-6. doi:10.1111/j.1365-2567.2008.02807.x

20. Bernardo L, Yu H, Amash A, Zimring JC, Lazarus AH. IgG-mediated immune suppression to erythrocytes by polyclonal antibodies can occur in the absence of activating or inhibitory Fc-gamma receptors in a full mouse model. J Immunol (2015) 195:2224-30. doi:10.4049/jimmunol.1500790

21. Bergström JJ, Heyman B. IgG suppresses antibody responses in mice lacking C1q, C3, complement receptors 1 and 2, or IgG Fc-receptors. PLoS One (2015) 10:e0143841. doi:10.1371/journal.pone.0143841

22. Heyman B, Wiersma E, Nose M. Complement activation is not required for IgG-mediated suppression of the antibody response. Eur J Immunol (1988) 18:1739-43. doi:10.1002/eji.1830181113

23. Daëron $\mathrm{M}$, Lesourne R. Negative signaling in Fc receptor complexes. $A d v$ Immunol (2006) 89:39-86. doi:10.1016/S0065-2776(05)89002-9

24. Na D, Kim D, Lee D. Mathematical modeling of humoral immune response suppression by passively administered antibodies in mice. J Theor Biol (2006) 241:830-51. doi:10.1016/j.jtbi.2006.01.019

25. Zhang Y, Meyer-Hermann M, George LA, Figge MT, Khan M, Goodall M, et al. Germinal center B cells govern their own fate via antibody feedback. $J$ Exp Med (2013) 210(3):457-64. doi:10.1084/jem.20120150

26. Takai T, Li M, Sylvestre D, Clynes R, Ravetch JV. FcRg chain deletion results in pleiotrophic effector cell defects. Cell (1994) 76:519-29. doi:10.1016/0092-8674(94)90115-5

27. Ey PL, Prowse SJ, Jenkin CR. Isolation of pure IgG1, IgG2a and IgG2b immunoglobulins from mouse serum using protein A-sepharose. Immunochemistry (1978) 15:429. doi:10.1016/0161-5890(78)90070-6

28. Heyman B, Brogren I, Klareskog L. Comparison between the sensitivity of the hemolytic plaque forming cell assay and the enzyme linked immunospot assay in detecting B-cells producing trinitrophenyl- or sheep erythrocyte specific IgM- and IgG antibodies. J Immunol Methods (1991) 139:293-6. doi:10.1016/0022-1759(91)90200-Y

29. Frey A, Di Canzio J, Zurakowski D. A statistically defined endpoint titer determination method for immunoassays. J Immunol Methods (1998) 221:35-41. doi:10.1016/S0022-1759(98)00170-7
30. Henningsson F, Ding Z, Dahlin JS, Linkevicius M, Carlsson F, Grönvik KO, et al. IgE-mediated enhancement of $\mathrm{CD} 4+\mathrm{T}$ cell responses in mice requires antigen presentation by CD11c+ cells and not by B cells. PLoS One (2011) 6:e21760. doi:10.1371/journal.pone.0021760

31. Stamm C, Barthelmann J, Kunz N, Toellner KM, Westermann J, Kalies K. Dose-dependent induction of murine Th1/Th2 responses to sheep red blood cells occurs in two steps: antigen presentation during second encounter is decisive. PLoS One (2013) 8:e67746. doi:10.1371/journal.pone. 0067746

32. Jacob J, Kelsoe G, Rajewsky K, Weiss U. Intraclonal generation of antibody mutants in germinal centres. Nature (1991) 354:389-92. doi:10.1038/ 354389a0

33. Kuraoka M, Schmidt AG, Nojima T, Feng F, Watanabe A, Kitamura $\mathrm{D}$, et al. Complex antigens drive permissive clonal selection in germinal centers. Immunity (2016) 44:542-52. doi:10.1016/j.immuni.2016. 02.010

34. Goodnow CC, Vinuesa CG, Randall KL, Mackay F, Brink R. Control systems and decision making for antibody production. Nat Immunol (2010) 11:681-8. doi:10.1038/ni.1900

35. Mesin L, Ersching J, Victora GD. Germinal center B cell dynamics. Immunity (2016) 45:471-82. doi:10.1016/j.immuni.2016.09.001

36. Weisel FJ, Zuccarino-Catania GV, Chikina M, Shlomchik MJ. A temporal switch in the germinal center determines differential output of memory B and plasma cells. Immunity (2016) 44:116-30. doi:10.1016/j.immuni.2015. 12.004

37. Finkelstein MS, Uhr JW. Specific inhibition of antibody formation by passively administered 19S and 7S antibody. Science (1964) 146:67-9. doi:10.1126/science.146.3640.67

38. Wigzell H. Antibody synthesis at the cellular level. Antibody-induced suppression of 7S antibody synthesis. J Exp Med (1966) 124:953-69. doi:10.1084/ jem.124.5.953

39. Chan PL, Sinclair NR. Regulation of the immune response. VI. Inability of $\mathrm{F}\left(\mathrm{ab}^{\prime}\right) 2$ antibody to terminate established immune responses and its ability to interfere with $\operatorname{IgG}$ antibody-mediated immunosuppression. Immunology (1973) 24:289-310.

40. Heyman B. Non-determinant specificity of feedback immunosuppression by IgG antibodies injected after the antigen. Scand J Immunol (1988) 27:361-5. doi:10.1111/j.1365-3083.1988.tb02358.x

41. Desmarets M, Cadwell CM, Peterson KR, Neades R, Zimring JC. Minor histocompatibility antigens on transfused leukoreduced units of red blood cells induce bone marrow transplant rejection in a mouse model. Blood (2009) 114:2315-22. doi:10.1182/blood-2009-04-214387

42. Yu H, Stowell SR, Bernardo L, Hendrickson JE, Zimring JC, Amash A, et al. Antibody-mediated immune suppression of erythrocyte alloimmunization can occur independently from red cell clearance or epitope masking in a murine model. J Immunol (2014) 193:2902-10. doi:10.4049/ jimmunol.1302287

43. Beliard R. Monoclonal anti-D antibodies to prevent alloimmunization: lessons from clinical trials. Transfus Clin Biol (2006) 13:58-64. doi:10.1016/j. tracli.2006.03.013

44. Kumpel BM. Lessons learnt from many years of experience using anti-D in humans for prevention of $\mathrm{RhD}$ immunization and haemolytic disease of the fetus and newborn. Clin Exp Immunol (2008) 154:1-5. doi:10.1111/j.1365-2249.2008.03735.x

45. Zarnitsyna VI, Lavine J, Ellebedy A, Ahmed R, Antia R. Multi-epitope models explain how pre-existing antibodies affect the generation of broadly protective responses to influenza. PLoS Pathog (2016) 12:e1005692. doi:10.1371/ journal.ppat.1005692

46. Brody NI, Walker JG, Siskind GW. Studies on the control of antibody synthesis. Interaction of antigenic competition and suppression of antibody formation by passive antibody on the immune response. J Exp Med (1967) 126:81-91. doi:10.1084/jem.126.1.81

47. Tao TW, Uhr JW. Capacity of pepsin-digested antibody to inhibit antibody formation. Nature (1966) 212:208-9. doi:10.1038/212208a0

48. Cerottini JC, McConahey PJ, Dixon FJ. The immunosuppressive effect of passively administered antibody IgG fragments. J Immunol (1969) 102: $1008-15$.

49. Heyman B. Antibody feedback suppression: towards a unifying concept? Immunol Lett (1999) 68:41-5. doi:10.1016/S0165-2478(99)00028-0 
50. Heyman B, Pilström L. Primary and secondary IgG antibodies are equally efficient immunosuppressors in relation to antigen binding capacity. Immunol Lett (1988) 17:189-94. doi:10.1016/0165-2478(88)90090-9

51. Quintana IZ, Silveira AV, Moller G. Regulation of the antibody response to sheep erythrocytes by monoclonal IgG antibodies. Eur J Immunol (1987) 17:1343-9. doi:10.1002/eji.1830170919

52. Bernardo L, Amash A, Marjoram D, Lazarus AH. Antibody-mediated immune suppression is effective when blends of anti-RBC monoclonal antibodies are used in mice. Blood (2016) 128:1076-80. doi:10.1182/ blood-2016-01-692178

53. Walker JG, Siskind GW. Studies on the control of antibody synthesis. Effect of antibody affinity upon its ability to suppress antibody formation. Immunology (1968) 14:21-8.

54. Woodrow JC, Clarke CA, Donohow WT, Finn R, McConnell RB, Sheppard PM, et al. Mechanism of Rh prophylaxis: an experimental study on specificity of immunosuppression. Br Med J (1975) 2:57-9. doi:10.1136/bmj.2. 5962.57

55. Gauthier E, Guo X, Mohandas N, An X. Phosphorylation-dependent perturbations of the 4.1R-associated multiprotein complex of the erythrocyte membrane. Biochemistry (2011) 50:4561-7. doi:10.1021/bi200154g

56. Junghans RP, Anderson CL. The protection receptor for IgG catabolism is the beta-2-microglobulin-containing neonatal intestinal transport receptor. Proc Natl Acad Sci U S A (1996) 93:5512-6. doi:10.1073/pnas.93. 11.5512

57. Siegrist CA. Mechanisms by which maternal antibodies influence infant vaccine responses: review of hypotheses and definition of main determinants. Vaccine (2003) 21:3406-12. doi:10.1016/S0264-410X(03)00342-6

58. Niewiesk S. Maternal antibodies: clinical significance, mechanism of interference with immune responses, and possible vaccination strategies. Front Immunol (2014) 5:446. doi:10.3389/fimmu.2014.00446

59. Edwards KM. Maternal antibodies and infant immune responses to vaccines. Vaccine (2015) 33:6469-72. doi:10.1016/j.vaccine.2015.07.085

Conflict of Interest Statement: The authors declare that the research was conducted in the absence of any commercial or financial relationships that could be construed as a potential conflict of interest.

Copyright (c) 2017 Bergström, Xu and Heyman. This is an open-access article distributed under the terms of the Creative Commons Attribution License (CC BY). The use, distribution or reproduction in other forums is permitted, provided the original author(s) or licensor are credited and that the original publication in this journal is cited, in accordance with accepted academic practice. No use, distribution or reproduction is permitted which does not comply with these terms. 